\title{
Règlementation relative à l'étiquetage et aux allégations des produits alimentaires en général, et des huiles et matières grasses en particulier
}

\author{
Morgane SAILLARD \\ FNCG, Fédération Nationale des Corps \\ Gras - Affaires Scientifiques et \\ Réglementaires, 66 rue La Boétie, \\ 75008 Paris \\ $<$ m.saillard@fncg.fr $>$
}

\begin{abstract}
European Regulation towards labelling and health claims made on foods in general, and vegetable oils and fats more specifically

Scientific research has evolved to identify relationship between nutrients and health effects. Theses scientific evidences can help to establish public health recommendations, which will be used by operators to formulate or optimize their food products. Labelling serves as a support for consumer information, raising its awareness about the food product nutritional composition. This enables the consumer to make an informed choice. Labelling also participates in the promotion of some of the food components, e.g. essential fatty acids in vegetable oils. In this context, regulation has evolved and allows operators to highlight nutritional benefits of their products via labelling information. Two different types of information must be distinguished: nutrition labelling and nutrition or health claims. The new European Regulation 1169/2011 on the provision of food information to consumers, published at the end of 2011, establishes a mandatory nutrition labelling with a fixed list of nutrients. Hence, food manufacturers are required to label the energy, fat, saturated fat, carbohydrate, sugars, protein and salt content of all their products. The Regulation on nutrition and health claims made on foods, published at the end of 2006, applies stringent rules to the use of claims and introduces the concept of a positive list for nutrition and generic health claims. This regulation aims at supporting public health recommendations and at providing the consumer with a high level of protection. A number of claims can be applied to vegetable oils. Nutrition labelling is an essential tool to disseminate and popularize scientific information in order to outreach consumers, as in the case of fatty acids, plant sterols or fat-soluble vitamins labeled in vegetable oils.
\end{abstract}

La recherche scientifique progresse pour mettre en évidence toujours plus de relations entre les nutriments et la santé. Ainsi, nos grands-parents nous faisaient consommerune cuillèreà souped'huilede foie de morue par semaine, sans que l'on sache nommer le nutriment bénéfique ou expliquer son mécanisme. Une chose était sûre: cela permettrait de nous garder en bonne santé. De même, le régime méditerranéen ou crétois est bien connu pour ses vertus. L'huile d'olive bénéficie ainsi d'une image nutritionnelle positive, sans que, pendant longtemps, les effets nutritionnels à l'œuvre aient été connus.
Désormais, les connaissances scientifiques ont évolué et les molécules présentant un intérêt nutritionnel sont précisément identifiées dans nos produits alimentaires : acide alphalinolénique, EPA, DHA, stérols végétaux... Toutefois, ces substances ne sont pas encore toutes bien connues du consommateur, même si elles commencent à apparaître sur les étiquetages des produits alimentaires.

Les recommandations de santé publique peuvent également alerter sur la présence de tel ou tel nutriment dont la consommation doit être limitée ; les consommateurs peuvent alors être en demande d'informations relatives à la présence ou la teneur de ce nutriment dans le produit qu'ils achètent.

Il est constaté une évolution positive des connaissances générales en matière de nutrition et santé, car selon l'enquête Comportements et Consommations Alimentaires des Français - CCAF, 2010 du CREDOC, $88 \%$ des consommateurs pensent que la manière dont ils mangent a une influence sur leur état de santé (contre $75 \%$ en 1997) (CCAF, 2010).

S'agissant des huiles végétales, la présence naturelle d'acides gras essen-

Pour citer cet article : Saillard M. Règlementation relative à l'étiquetage et aux allégations des produits alimentaires en général, et des huiles et matières grasses en particulier. OCL $2012 ; 19(2)$ : 76-82. doi : 10.1684 /ocl.2012.0439 
tiels est maintenant bien connue de la communauté scientifique, et commence également à l'être des consommateurs. Selon une étude CREDOC réalisée en 2011, 82 \% des consommateurs savent que les huiles végétales contiennent des acides gras essentiels (Credoc, 2011). Toutefois la proportion des consommateurs ayant une connaissance plus détaillée des acides gras (types, fonctions. . .) diminue considérablement.

Parmi les nutriments bénéfiques pour la santé, certaines vitamines liposolubles ou les stérols végétaux présents dans les huiles végétales restent également méconnus à ce jour.

Les étiquetages des produits mentionnent depuis de nombreuses années la présence d'oméga 3,6 ou 9 , ou bien plus récemment font état $d^{\prime}$ 'une relation plus complexe entre ces substances et une fonction santé.

Malgré les progrès réalisés en matière d'information et d'éducation du consommateur, les efforts doivent être maintenus sur la sensibilisation à la nutrition et à l'importance d'une alimentation équilibrée et diversifiée. C'est pourquoi l'éducation reste l'un des axes majeurs de la politique de santé publique en France, dans I'objectif d'induire un comportement rationnel de la part du consommateur grâce à l'apport d'informations pouvant influencer la perception risque/bénéfice d'un produit alimentaire (INRA-ESCo, 2010).

Dans ce contexte, la réglementation a récemment évolué pour permettre aux opérateurs de valoriser la présence des nutriments contenus dans les denrées alimentaires, dans le but d'améliorer l'information du consommateur, mais également pour encadrer les mentions figurant sur les étiquetages.

Deux réglementations ont vu le jour :

- le règlement (CE) no 1924/2006 relatif aux allégations nutritionnelles et de santé ;

- le règlement (UE) $n^{\circ} 1169 / 2011$ concernant l'information du consommateur sur les denrées alimentaires.

\section{Les allégations nutritionnelles et de santé}

Le règlement (CE) n¹924/2006 (Union européenne, 2006a), adopté le
20 décembre 2006, définit les allégations nutritionnelles et de santé, et encadre leur utilisation. Auparavant, il n'existait pas de législation régissant I'utilisation des allégations. En France, la vérification s'effectuait a posteriori, après contrôle des étiquetages, et il était demandé à l'opérateur de justifier son allégation sur la base d'éléments scientifiques.

De manière à harmoniser les allégations présentes sur les emballages en évitant toute distorsion de concurrence, et à assurer au consommateur un niveau élevé de protection, la Commission a donc décidé d'élaborer un règlement communautaire relatif aux allégations.

Un système de profils nutritionnels pour identifier les produits alimentaires éligibles aux allégations a été initialement prévu par le règlement (article 4 du règlement). Cependant, la Commission $\mathrm{n}^{\prime}$ a toujours pas mis en place ce système au vu de la difficulté à élaborer des critères nutritionnels sélectifs pour les différentes catégories de denrées alimentaires.

Le règlement distingue deux types d'allégations :

- les allégations nutritionnelles qui affirment, suggèrent ou impliquent qu'une denrée alimentaire possède des propriétés nutritionnelles bénéfiques particulières par l'énergie ou les nutriments qu'elle apporte, n'apporte pas ou apporte en proportion moindre ou plus élevée (article 2 du règlement) ;

- les allégations de santé, qui affirment, suggèrent ou impliquent l'existence d'une relation entre, d'une part, une catégorie de denrées alimentaires, une denrée alimentaire ou l'un de ses composants, et d'autre part, la santé (article 2 du règlement).

Parmi les allégations de santé on distingue deux sous-catégories :

a) les allégations de santé génériques (article 13 du règlement);

b) les allégations de santé relatives à la réduction d'un risque de maladie ou se rapportant au développement et à la santé de l'enfant (article 14 du règlement).

Le règlement encadre de manière stricte I'utilisation des allégations, car il introduit la notion de liste positive d'allégations, et impose des critères quantitatifs et qualitatifs sur la substance faisant l'objet de l'allégation ainsi que sur la matrice produit. Ceci toujours dans un but de protection élevée du consommateur, et de lui assurer la disposition de produits convenablement étiquetés. Une validation officielle et réglementaire est requise avant toute utilisation d'une allégation par les opérateurs.

Une allégation ne pourra être utilisée que si :

- le nutriment ou la substance :

- a un effet nutritionnel ou physiologique bénéfique reconnu,

- se trouve dans le produit en quantité significative, ou son absence/sa réduction permet de produire l'effet affirmé,

- se trouve dans une forme permettant à I'organisme de l'utiliser ;

- la quantité de produit susceptible d'être consommée apporte bien une quantité significative de nutriment/substance.

Pour toutes les allégations de santé, le produit doit par ailleurs comporter une mention sur l'importance d'une alimentation variée et équilibrée et d'un mode de vie sain. D'autre part une allégation ne peut pas faire référence au rythme ou à l'importance d'une perte de poids.

Les allégations nutritionnelles autorisées sont listées en annexe du règlement : " source de ", " riche en ", " faible teneur en ", etc.

Les allégations de santé génériques font elles aussi l'objet d'une liste positive actuellement en cours de validation : suite aux avis scientifiques d'évaluation de I'AESA, elles ont été introduites dans un projet de règlement par la Commission. A la date de rédaction de cet article, la liste des allégations de santé génériques qui seront autorisées dans I’Union Européenne a été validée par le Conseil européen; elle est en cours d'examen par le Parlement européen.

Les allégations de santé relevant de l'article 14 (pour rappel : allégations de santé relatives à la réduction d'un risque de maladie ou se rapportant au développement et à la santé de l'enfant) font l'objet d'une procédure différente : un dossier particulier doit être remis par l'opérateur à I'AESA via son autorité nationale. Une fois I'avis rendu, la Commission fait paraître un règlement autorisant ou rejetant l'allégation demandée.

L'utilisation d'une allégation doit être accompagnée de l'indication de la teneur en nutriment allégué dans le tableau de valeur nutritionnel (article 7 du règlement). 
Les allégations nutritionnelles figurant dans l'annexe du Règlement (CE) $n^{\circ} 1924 / 2006$ et susceptibles d'êtres utilisées pour les huiles végétales sont listées dans le tableau 1

Les allégations de santé génériques susceptibles d'être utilisées pour les huiles végétales et figurant à ce jour dans le projet de règlement sont listées dans le tableau 2.

Les huiles végétales peuvent utiliser ces allégations pour mettre en avant leurs caractéristiques nutritionnelles, différentes selon le type d'huile. Les produits contenant de I'huile végétale peuvent également prétendre à des allégations en fonction de la quantité d'huile incorporée dans le produit fini.

À titre d'exemple, pour I'utilisation de l'allégation de santé générique "L'acide alphalinolénique (ALA) contribue au maintien d'une cholestérolémie normale » par une huile végétale, les conditions suivantes devraient êtres remplies :

- I'huile doit contenir au moins 0,3 g $\mathrm{d}^{\prime} \mathrm{ALA} / 100 \mathrm{~g}$ et $100 \mathrm{kcal}$, soit $0,3 \mathrm{~g}$ d'ALA pour 11, $1 \mathrm{~g}$ d'huile vu la densité énergétique de l'huile ;

- il doit être mentionné sur l'étiquetage que l'effet bénéfique est obtenu par la consommation journalière de $2 \mathrm{~g}$ d'ALA ;

- il doit apparaître sur l'étiquetage une mention sur l'importance d'une alimentation variée et équilibrée et d'un mode de vie sain ;

- la quantité d'oméga-3 ALA doit être étiquetée dans le tableau de valeur nutritionnel, par exemple sous la forme représentée dans le tableau 3 (ce point reste à confirmer).

La profession de I'huilerie a défini au niveau européen la portion d'huile à $10 \mathrm{~g}$, soit une cuillère à soupe.

Les différentes allégations autorisées ont pour but d'accompagner les recommandations de santé publique (notion de pertinence nutritionnelle utilisée par la Commission), et d'inciter à la reformulation des produits en fonction des besoins nutritionnels de la population. A titre d'exemple, les États membres ont refusé l'utilisation d'une allégation relative aux lipides et à l'absorption des vitamines liposolubles ayant pourtant reçu un avis positif de I'AESA, car ils considèrent qu'elle peut entraîner la confusion dans l'esprit du consommateur au vu des messages de santé publique qui demandent globalement la diminution des apports en lipides.
Même $s^{\prime}$ il n'est pas facile de pouvoir évaluer les besoins à l'échelle européenne de par des comportements alimentaires différents dans les États membres, I'AESA a travaillé sur des apports de référence communautaires. Elle a publié en 2010 des valeurs nutritionnelles de référence pour les lipides, les AGS, les AGPI, les AGMI, les AGT et le cholestérol (EFSA, 2010). Ces recommandations identifient la contribution conseillée des différents nutriments à l'apport énergétique journalier. II est à noter que les recommandations européennes et nationales ne concordent pas toujours (tableau 4).

Une fois retranscrites sur l'étiquetage du produit en termes de pourcentage d'apport de référence, elles doivent permettre au consommateur d'évaluer et d'équilibrer au mieux son apport nutritionnel.

\section{L'information du consommateur par I'étiquetage nutritionnel}

L'étiquetage des denrées alimentaires doit être clair et compréhensible pour le consommateur, afin de lui fournir toutes

Tableau 1. Exemple d'allégations nutritionnelles répertoriées dans l'annexe du règlement (CE) n¹924/2006

\begin{tabular}{|c|c|}
\hline Allégation nutritionnelle & Conditions d'utilisation \\
\hline $\begin{array}{l}\text { Source de [nom de vitamines et/ou minéraux]. } \\
\text { Ex : vitamines liposolubles }\end{array}$ & $\begin{array}{l}\text { Le produit contient au moins la quantité significative définie à l'annexe de la } \\
\text { directive 90/496/CEE ou une quantité prévue au titre de dérogations accordées } \\
\text { conformément à l'article } 6 \text { du règlement (CE) nº1925/2006 (Union européenne, } \\
\text { 2006b). }\end{array}$ \\
\hline $\begin{array}{l}\text { Riche en [nom de vitamines et/ou minéraux]. } \\
\text { Ex : vitamine liposolubles }\end{array}$ & $\begin{array}{l}\text { Le produit contient au moins deux fois la teneur requise pour l'allégation "source } \\
\text { de [nom de vitamines et/ou minéraux]". }\end{array}$ \\
\hline Contient [nom de vitamines et/ou minéraux] & $\begin{array}{l}\text { Le produit respecte toutes les dispositions applicables du présent règlement, } \\
\text { et notamment l'article } 5 \text {. Pour les vitamines et minéraux, les conditions prévues } \\
\text { pour l'allégation " source de " s'appliquent. }\end{array}$ \\
\hline $\begin{array}{l}\text { Enrichi en [nom de vitamines et/ou minéraux]. } \\
\text { Ex : vitamines liposolubles }\end{array}$ & $\begin{array}{l}\text { Le produit remplit les conditions applicables à l'allégation "source de " } \\
\text { et l'augmentation de cette teneur est d'au moins } 30 \% \text { par rapport à } \\
\text { un produit similaire. }\end{array}$ \\
\hline Source d'acide gras oméga-3 & $\begin{array}{l}\text { Le produit contient au moins } 0,3 \mathrm{~g} \text { d'acide alphalinolénique pour } 100 \mathrm{~g} \text { et } 100 \mathrm{kcal} \text {, } \\
\text { ou au moins } 40 \mathrm{mg} \text { d'acide eicosapentaenoïque et d'acide docosahexanoïque } \\
\text { combinés pour } 100 \mathrm{~g} \text { et } 100 \mathrm{kcal} \text {. }\end{array}$ \\
\hline Riche en acide gras oméga- 3 & $\begin{array}{l}\text { Le produit contient au moins } 0,6 \mathrm{~g} \text { d'acide alphalinolénique pour } 100 \mathrm{~g} \text { et } 100 \mathrm{kcal} \text {, } \\
\text { ou au moins } 80 \mathrm{mg} \text { d'acide eicosapentaenoïque et d'acide docosahexanoïque } \\
\text { combinés pour } 100 \mathrm{~g} \text { et } 100 \mathrm{kcal} \text {. }\end{array}$ \\
\hline Riche en graisses mono-insaturées & $\begin{array}{l}\text { Le produit contient au moins } 45 \% \text { d'acides gras dérivés de graisses } \\
\text { mono-insaturées et l'énergie fournie par les graisses mono-insaturées représente } \\
\text { plus de } 20 \% \text { de l'apport énergétique du produit. }\end{array}$ \\
\hline Riche en graisses polyinsaturées & $\begin{array}{l}\text { Le produit contient au moins } 45 \% \text { d'acides gras dérivés de graisses } \\
\text { polyinsaturées et l'énergie fournie par les graisses polyinsaturées représente } \\
\text { plus de } 20 \% \text { de l'apport énergétique du produit. }\end{array}$ \\
\hline
\end{tabular}


Tableau 2. Exemple d'allégations de santé génériques figurant dans le projet de règlement de la Commission.

\begin{tabular}{|c|c|}
\hline Allégation de santé générique & Conditions d'utilisation \\
\hline $\begin{array}{l}\text { L'acide alphalinolénique (ALA) contribue au maintien d'une } \\
\text { cholestérolémie normale }\end{array}$ & $\begin{array}{l}\text { La denrée alimentaire est au moins une source d'ALA au sens } \\
\text { de l'allégation " source d'acide gras oméga- } 3 \text { " définie dans } \\
\text { l'annexe du règlement (CE) n } n^{\circ} 1924 / 2006 \text {. } \\
\text { Le consommateur doit être informé que l'effet bénéfique est } \\
\text { obtenu par la consommation journalière de } 2 \text { g d'ALA. }\end{array}$ \\
\hline $\begin{array}{l}\text { L'acide docosahexaénoïque (DHA) contribue au fonctionnement } \\
\text { normal du cerveau }\end{array}$ & $\begin{array}{l}\text { La denrée alimentaire contient au moins } 40 \mathrm{mg} \text { d'acide } \\
\text { docosahexaénoïque (DHA) pour } 100 \mathrm{~g} \text { et pour } 100 \mathrm{kcal} \text {. } \\
\text { L'allégation peut être utilisée si le consommateur est informé } \\
\text { que l'effet bénéfique est obtenu par la consommation } \\
\text { journalière de } 250 \mathrm{mg} \text { de DHA. }\end{array}$ \\
\hline $\begin{array}{l}\text { L'acide docosahexaénoïque (DHA) contribue au maintien d'une } \\
\text { vision normale }\end{array}$ & $\begin{array}{l}\text { La denrée alimentaire contient au moins } 40 \mathrm{mg} \text { de DHA pour } \\
100 \mathrm{~g} \text { et pour } 100 \mathrm{kcal} \text {. L'allégation peut être utilisée si le } \\
\text { consommateur est informé que l'effet bénéfique est obtenu } \\
\text { par la consommation journalière de } 250 \mathrm{mg} \text { de DHA. }\end{array}$ \\
\hline $\begin{array}{l}\text { L'acide eicosapentaénoïque (EPA) et l'acide docosahexaénoïque } \\
\text { (DHA) contribuent à une fonction cardiaque normale }\end{array}$ & $\begin{array}{l}\text { La denrée alimentaire est au moins une source d'EPA et de } \\
\text { DHA au sens de l'allégation " source d'acide gras oméga-3 " } \\
\text { définie dans l'annexe du règlement (CE) n } 1924 / 2006 \text {. } \\
\text { L'allégation peut être utilisée si le consommateur est informé } \\
\text { que l'effet bénéfique est obtenu par la consommation } \\
\text { journalière de } 250 \text { mg d'EPA et de DHA. }\end{array}$ \\
\hline $\begin{array}{l}\text { La réduction de la consommation de graisses saturées contribue } \\
\text { au maintien d'une cholestérolémie normale }\end{array}$ & $\begin{array}{l}\text { La denrée alimentaire dont la teneur en acides gras saturés } \\
\text { est au moins faible au sens de l'allégation « faible teneur } \\
\text { en graisses saturées " } \\
\text { ou réduite au sens de l'allégation } \\
\text { "réduit en [nom du nutriment] " définies dans l'annexe du } \\
\text { règlement (CE) n 1924/2006. }\end{array}$ \\
\hline $\begin{array}{l}\text { L'acide linoléique contribue au maintien d'une cholestérolémie } \\
\text { normale }\end{array}$ & $\begin{array}{l}\text { La denrée alimentaire fournit au moins } 1,5 \mathrm{~g} \text { d'acide linoléique } \\
\text { pour } 100 \mathrm{~g} \text { et } 100 \mathrm{kcal} \text {. } \\
\text { Le consommateur doit être informé que l'effet bénéfique } \\
\text { est obtenu par la consommation journalière de } 10 \mathrm{~g} \text { d'acide } \\
\text { linoléique. }\end{array}$ \\
\hline $\begin{array}{l}\text { Le remplacement de graisses saturées par des graisses insaturées } \\
\text { dans le régime alimentaire contribue au maintien d'une } \\
\text { cholestérolémie normale [les acides gras mono-insaturés (AGMI) } \\
\text { et les acides gras polyinsaturés (AGPI) sont des graisses insaturées] }\end{array}$ & $\begin{array}{l}\text { La denrée alimentaire est riche en acides gras insaturés au } \\
\text { sens de l'allégation " riche en graisses insaturées » définie } \\
\text { dans l'annexe du règlement (CE) n 1924/2006. }\end{array}$ \\
\hline $\begin{array}{l}\text { Le remplacement de graisses saturées par des graisses insaturées } \\
\text { dans le régime alimentaire contribue au maintien d'une } \\
\text { cholestérolémie normale. L'acide oléique est une graisse insaturée. }\end{array}$ & $\begin{array}{l}\text { La denrée alimentaire est riche en acides gras insaturés au sens } \\
\text { de l'allégation " riche en graisses insaturées " définie dans } \\
\text { l'annexe du règlement (CE) n 1924/2006. }\end{array}$ \\
\hline $\begin{array}{l}\text { Les stérols végétaux/stanols végétaux contribuent au maintien } \\
\text { d'une cholestérolémie normale }\end{array}$ & $\begin{array}{l}\text { L'allégation peut être utilisée si le consommateur est informé } \\
\text { que l'effet bénéfique est obtenu par la consommation } \\
\text { journalière d'au moins } 0,8 \mathrm{~g} \text { de stérols végétaux/stanols végétaux. }\end{array}$ \\
\hline $\begin{array}{l}\text { Les polyphénols présents dans I'huile d'olive contribuent à } \\
\text { protéger les lipides sanguins contre le stress oxydatif }\end{array}$ & $\begin{array}{l}\text { L'huile d'olive contient au moins } 5 \mathrm{mg} \text { d'hydroxytyrosol et } \\
\text { ses dérivés (comme le complexe oleuropéine et le tyrosol) } \\
\text { pour } 20 \mathrm{~g} \text { d'huile d'olive. L'allégation peut être utilisée si le } \\
\text { consommateur est informé que l'effet bénéfique est obtenu } \\
\text { par la consommation journalière de } 20 \mathrm{~g} \text { d'huile d'olive. }\end{array}$ \\
\hline
\end{tabular}

les informations nécessaires pour choisir les denrées qu'il consomme. Les informations fournies peuvent être diverses: composition du produit, mode d'emploi, date limite de consommation, information nutritionnelle, présence potentielle d'allergènes, etc. Tous ces éléments doivent être transmis le plus lisiblement possible au consommateur, tout en laissant à l'opérateur la possibilité de mettre en avant les qualités de son produit qu'il jugera importantes.

Les différents textes relatifs à l'étiquetage général, à sa lisibilité, à l'étiquetage nutritionnel ou encore à la présence $\mathrm{d}^{\prime}$ allergènes ont donc été rassemblés en un seul texte européen. Ce texte introduit également de nouvelles dispositions pour assurer un niveau de protection toujours plus élevé pour le consommateur.

\section{Les principales dispositions du règlement relatif à} I'information du consommateur

Le règlement (UE) $n^{\circ} 1169 / 2011$ (Union européenne, 2011) concernant l'information du consommateur a été 
Tableau 3. Possible représentation (qui doit néanmoins être confirmée) du tableau nutritionnel d'une huile végétale, souhaitant revendiquer l'allégation de santé générique "L'acide alphalinolénique (ALA) contribue au maintien d'une cholestérolémie normale "et devant, à ce titre, mentionner la quantité d'oméga-3 ALA dans ledit tableau.

\begin{tabular}{|c|c|c|}
\hline Valeurs nutritionnelles & Pour $100 \mathrm{~g}$ & $\begin{array}{l}\text { Pour une portion } \\
\text { de } 10 \mathrm{~g}\end{array}$ \\
\hline Energie & $\mathrm{X}$ kcal & X Kcal \\
\hline Graisses & X g & $\mathrm{Xg}$ \\
\hline \multicolumn{3}{|l|}{ dont } \\
\hline Acides gras saturés & $\mathrm{Xg}$ & $\mathrm{Xg}$ \\
\hline Acides gras mono-insaturés & $\mathrm{Xg}$ & $\mathrm{Xg}$ \\
\hline Acides gras polyinsaturés & $\mathrm{Xg}$ & $\mathrm{Xg}$ \\
\hline dont oméga-3 ALA & $\mathrm{Xg}$ & $\mathrm{Xg}$ \\
\hline Glucides & $0 \mathrm{~g}$ & $0 \mathrm{~g}$ \\
\hline dont sucres & $0 \mathrm{~g}$ & $0 \mathrm{~g}$ \\
\hline Protéines & $0 \mathrm{~g}$ & $0 \mathrm{~g}$ \\
\hline Sel & $0 \mathrm{~g}$ & $0 \mathrm{~g}$ \\
\hline
\end{tabular}

adopté le 25 octobre 2011, après presque quatre ans de discussions entre la Commission européenne, le Parlement européen et le Conseil. Les échanges ont été nombreux entre les trois instances en raison de divergence sur des sujets clés du règlement, comme l'étiquetage nutritionnel, l'étiquetage de l'origine ou encore les critères de lisibilité.

Ce règlement remplace tous les textes relatifs à l'étiquetage des denrées alimentaires, et instaure notamment de nouvelles dispositions pour l'étiquetage nutritionnel. En effet, I'ancienne directive 90/496/CEE est abrogée et remplacée par les dispositions des articles 9 et 29 à 35 du règlement. Le règlement impose, contrairement à la directive, une application immédiate par tous les États membres sans passer par l'étape de transposition par les autorités nationales.

Selon I'article 9, point I du règlement, la déclaration nutritionnelle devient obligatoire pour toutes les denrées alimentaires (sauf exceptions énumérées à l'article 16 du règlement). Les huiles végétales ne dérogent pas à la règle, et doivent présenter sur leur étiquetage un tableau de valeur nutritionnelle reprenant l'ensemble des nutriments concernés par l'étiquetage nutritionnel. Cette pratique était par ailleurs déjà largement répandue parmi les industriels du secteur, d'autant plus que le règlement (CE) $n^{\circ} 1924 / 2006$ concernant les allégations nutritionnelles et de santé obligeait déjà en cas d'allégation à préciser la teneur du nutriment concerné.

Les nutriments à étiqueter de manière obligatoire pour $100 \mathrm{~g}$ de produit figurent dans le tableau 5.

Une seconde liste fixe les nutriments pouvant être indiqués de façon volontaire (tableau 6).

Cette déclaration nutritionnelle doit apparaître dans le même champ visuel du produit, définit comme " toutes les surfaces d'un emballage pouvant être lues à partir d'un unique angle de vue".

Une répétition des informations nutritionnelles sur le champ visuel principal de l'emballage reste néanmoins possible, sous réserve qu'il s'agisse :

- soit de la seule valeur énergétique ; - soit de la valeur énergétique, des graisses, des acides gras saturés, des sucres et du sel.

Rappelons que le champ visuel principal se définit comme "le champ visuel d'un emballage le plus susceptible d'être vu au premier coup d'œil par les consommateurs lors de l'achat et permettant à ces derniers d'identifier immédiatement un produit en fonction de ses caractéristiques et de sa nature; [...] si un emballage comporte plusieurs champs visuels identiques, le champ visuel principal est celui choisi par l'exploitant du secteur alimentaire ".

Enfin, le pourcentage des apports de référence apportés par la portion peut être indiqué, les apports de référence pour les principaux nutriments étant fixés par I'annexe XIII, partie B du règlement. L'Agence européenne de

Tableau 4. AESA 2010 - Valeurs de référence pour les lipides et acides gras, et le cholestérol.

\begin{tabular}{|lll|}
\hline & Apport de référence (adulte) & \\
\hline & Valeurs de référence européennes AESA 2010 & ANC français 2010 \\
\hline Lipides & $20-35 \%$ de l'apport énergétique $(\mathrm{E})$ & $35-40 \%$ de l'apport énergétique \\
\hline AGS & Aussi bas que possible & $<12 \% \mathrm{E}$ \\
\hline Dont C12:0, C14:0 et C16:0 & & $<8 \% \mathrm{E}$ \\
\hline AGMI & & $15-20 \% \mathrm{E}$ \\
\hline Dont acide oléique & & $1 \% \mathrm{E}$ \\
\hline AGPI oméga-3 & $0,5 \% \mathrm{E}$ & $250 \mathrm{mg}$ \\
\hline Dont ALA & $250 \mathrm{mg}$ & $4 \% \mathrm{E}$ \\
\hline Dont EPA/DHA & $4 \% \mathrm{E}$ & \\
\hline AGPI oméga-6 & Aussi bas que possible & \\
\hline AGT &
\end{tabular}


Tableau 5. Nutriments d'étiquetage obligatoire.

\begin{tabular}{|ll|}
\hline Nutriment & Unité d'expression \\
\hline Energie & $\mathrm{kJ} / \mathrm{kcal}$ \\
\hline graisses & $\mathrm{g}$ \\
\hline acides gras saturés & $\mathrm{g}$ \\
\hline glucides & $\mathrm{g}$ \\
\hline sucres & $\mathrm{g}$ \\
\hline protéines & $\mathrm{g}$ \\
\hline
\end{tabular}

sécurité alimentaire (AESA) a également publié des valeurs de références pour l'étiquetage pour les oméga- 3 et oméga-6 (EFSA, 2009).

Ce modèle de présentation « à la portion " est déjà utilisé de manière volontaire par un grand nombre d'entreprises européennes et recommandé par la fédération européenne des industries alimentaires FoodDrinkEurope (CIAA, 2010) dans une volonté d'harmonisation des pratiques: les pourcentages des repères nutritionnels journaliers pour un adulte apportés par une portion de produit sont indiqués sous forme de cartouche identifiable sur l'emballage.

\section{L'application pour les huiles végétales}

La réglementation permet donc aux opérateurs d'apporter des précisions sur les teneurs en lipides (terme " graisses " repris dans le règlement) et différents acides gras, ainsi qu'en vitamines liposolubles et stérols végétaux des huiles végétales et des produits en contenant. Ces informations factuelles transmises au consommateur doivent lui permettre de faire ses choix en toute connaissance de cause.

La réglementation encourage également la substitution ou la sélection pour réduire ou limiter la présence de nutriments dont les pouvoirs publics recom-

Tableau 6. Nutriments d'etiquetage volontaire

\begin{tabular}{|ll|}
\hline Nutriment & Unité \\
\hline Acides gras mono-insaturés & $\mathrm{g}$ \\
\hline Acides gras polyinsaturés & $\mathrm{g}$ \\
\hline Polyols & $\mathrm{g}$ \\
\hline Amidon & $\mathrm{g}$ \\
\hline Fibres alimentaires & $\mathrm{g}$ \\
\hline
\end{tabular}

mandent de limiter la consommation. C'est par exemple le cas des acides gras saturés (AGS) ou des acides gras trans (AGT), selon les nouveaux apports nutritionnels conseillés français pour les acides gras (AFSSA, 2010).

Au niveau européen les AGT doivent faire l'objet d'un rapport d'étude de la Commission dans les trois ans suivant la publication du règlement de 2011 (article 30 paragraphe 7 du règlement), soit d'ici 2014. La Commission doit en effet évaluer leur présence dans les denrées alimentaires, et statuer sur la nécessité ou non de prendre des mesures spécifiques: étiquetage obligatoire, limitation des teneurs, etc. Elle interrogera pour cela les différents secteurs alimentaires pour établir un état des lieux de la situation des AGT en Europe.

$S^{\prime}$ agissant des huiles végétales, la teneur moyenne en AGT se situe à $0,47 \mathrm{~g} /$ $100 \mathrm{~g}$, et elles contribuent entre 0,7 et $0,8 \%$ des apports totaux en AGT de la population française (AFSSA, 2009).

En attendant la publication ce rapport, les AGT ne peuvent plus être étiquetés, même de manière volontaire, sur les produits.

Les huiles végétales ont naturellement une grande proportion d'acides gras insaturés, ce quileur confère leur fluidité : huile de colza, huile de tournesol, huile d'olive, etc. Néanmoins certaines huiles plus riches en acides gras saturés présentent à température ambiante des textures concrètes, comme c'est le cas pour I'huile de coprah ou I'huile de palme. L'utilisateur d'huile végétale devra donc prendre en compte à la fois les composantes nutritionnelles et fonctionnelles des huiles, en fonction de I'application et du produit de destination. La richesse en acides gras insaturés des huiles végétales, notamment les acides gras essentiels que sont l'acide alphalinolénique (oméga-3) et l'acide linoléique (oméga-6), peut être mise en avant sur l'étiquetage nutritionnel du produit. La forme d'étiquetage reste à confirmer, puisque le règlement mentionne les acides gras polyinsaturés (AGPI) sans citer précisément les oméga-3.

La présence d'une allégation relative aux oméga-3 oblige cependant l'opérateur à indiquer la teneur en oméga-3 de I'huile dans le tableau de valeurs nutritionnelles.

La forme de la déclaration nutritionnelle est fixée par le règlement de 1169/
2011, notamment l'ordre dans lequel les nutriments doivent apparaître.

Contrairement à l'ancienne directive, ce règlement oblige, même en l'absence d'allégations, à indiquer la teneur en nutriments de manière exhaustive selon la liste de nutriments obligatoires ( $c$ 'està-dire sel, sucres...), ce qui est peu pertinent pour les huiles végétales qui contiennent $100 \%$ de lipides. Cette évolution traduit la volonté d'harmonisation des informations mises à disposition du consommateur, pour faciliter la lisibilité et les comparaisons entre les denrées alimentaires.

\section{Conclusion}

Les recommandations de santé publique doivent s'accompagner de réalisations concrètes pour sensibiliser les consommateurs à la lecture et à la compréhension des informations nutritionnelles qui lui sont présentées. L'étiquetage nutritionnel est un outil indispensable qui participe à la diffusion et à la vulgarisation de l'information scientifique disponible. L'actualisation de la réglementation amorce cette démarche et permet, par exemple, la valorisation des différents constituants des huiles végétales auprès du consommateur.

Enfin, si un focus est fait ici sur la réglementation et la mise en avant des qualités nutritionnelles du produit, l'aspect "plaisir" en doit pas être oublié car il est essentiel en matière d'alimentation.

\section{RÉFÉRENCES}

CCAF. Enquête Comportements et Consommations Alimentaires des Français - CCAF, 2010.

Credoc. Etude CREDOC pour I'ONIDOL Etude sur la perception et l'usage des huiles végétales. Octobre 2011.

INRA-ESCo. Les comportements alimentaires. Juin 2010.

Union européenne. Règlement (UE) n¹169/ 2011 du Parlement Européen et du Conseil du 25 octobre 2011 concernant l'information du consommateur. JOUE du 22/11/2011.

EFSA. Scientific Opinion of the Panel on Dietetic products, Nutrition and Allergies on a request from European Commission related to labelling reference intake values for $n-3$ and $n-6$ polyunsaturated fatty acids. The EFSA Journal $2009 ;(1176)$ : 1-11. 
CIAA. Nutrition Labeling: Understanding the information. http://gda.ciaa.eu/documents/ others $\$ /$ Final-brochure.pdf.

AFSSA. Avis de l'Agence française de sécurité sanitaire des aliments relatif à l'actualisation des apports nutritionnels conseillés pour les acides gras - Saisine $n^{\circ} 2006-S A-0359$. Mars 2010.

AFSSA. Avis de l'Agence française de sécurité sanitaire des aliments sur l'estimation des apports en acides gras trans de la population française - Saisine $n^{\circ}$ 2007-SA-0220. Février 2009.

Union européenne. Règlement (CE) n¹924/ 2006 du Parlement Européen et du Conseil du 20 décembre 2006 concernant les allégations nutritionnelles et de santé portant sur les denrées alimentaires. JOUE du 30/12/2006.

Union européenne. Règlement (CE) n¹925/ 2006 du Parlement européen et du Conseil du 20 décembre 2006 concernant I'adjonction de vitamines, de minéraux et de certaines autres substances aux denrées alimentaires.

EFSA. EFSA Panel on Dietetic Products, Nutrition, and Allergies (NDA); Scientific Opinion on Dietary Reference Values for fats, including saturated fatty acids, polyunsaturated fatty acids, monounsaturated fatty acids, trans fatty acids, and cholesterol. EFSA Journal $2010 ; 8: 1461$. 